\title{
AURAL POLYPS: WHAT'S BEHIND THEM?
}

\author{
Alice Lang Silva ${ }^{1}$, Fábio André Selaimen ${ }^{1}$, Isabel Saorin Conte ${ }^{2}$, \\ Marcela Lehmkuhl Damiani ${ }^{1}$, Luciana Lima Martins Costa ${ }^{1}$, \\ Letícia Petersen Schmidt Rosito ${ }^{1}$, Sady Selaimen da Costa ${ }^{1}$ \\ ${ }^{1}$ Hospital de Clínicas de Porto Alegre and Department of Ophthalmology and \\ Otorhinolaryngology, Faculty of Medicine, Universidade Federal do Rio Grande do Sul, \\ Porto Alegre, RS, Brazil \\ ${ }^{2}$ Universidade Federal do Rio Grande do Sul, Porto Alegre, RS, Brazil
}

\begin{abstract}
INTRODUCTION: Polyps in the external auditory canal (EAC) may be present due to inflammation induced by chronic otitis media. In many cases, the type of underlying otitis media is a cholesteatoma.

AIM: The aim of this article is to determine the etiology of EAC polyps in patients referred to the Otology Division of a tertiary hospital in south Brazil.

MATERIALS AND METHODS: Cross-sectional study was conducted with a cohort of patients with otologic diseases who were evaluated from July 2001 to December 2020. Patients with unilateral or bilateral aural polyps underwent anamnesis and recorded video-otoscopy and had their online medical chart reviewed.

RESULTS: Of the 2432 patients evaluated, $133(5.4 \%)$ had a polyp in the external auditory canal. Of those, 53 were excluded, leaving a final sample of 81 patients (3.3\%).

DISCUSSION: Middle ear cholesteatoma (MEC) and non-cholesteatomatous chronic otitis media (NCCOM) were responsible for $\mathbf{8 6 . 3 \%}$ of all polyps evaluated and the majority of diagnoses were established through surgery $(\mathbf{7 6 . 5 \% )}$.
\end{abstract}

CONCLUSION: Symptoms associated with polyps and their aspect do not reveal the most probable etiologies making imaging exams, biopsy and surgery necessary steps in aural polyp investigation.

Keywords: cholesteatoma, polyp, external ear

Address for correspondence:

Alice Lang Silva

Department of Otorhinolaryngology

Hospital de Clínicas de Porto Alegre

Rua Ramiro Barcelos 2350

90035-903 Porto Alegre, RS

Brazil

e-mail:alicelangsilva@gmail.com

Received: May 22, 2021

Accepted: June 13, 2021

\section{INTRODUCTION}

The finding of a polyp in the external auditory canal (EAC) is often thought to be a manifestation of inflammatory middle ear disease. Also known as aural polyp (AP), it is usually characterized as a soft to rubbery reddish mass (1) within the external auditory canal, therefore, lateral to the tympanic membrane.

The type of chronic otitis media (COM) that first comes to mind for most otologists as the under- 
lying cause for AP is cholesteatoma. Previous studies in the last decades estimated a great variability of cholesteatoma as the final diagnosis for polyps, with prevalence ranging from $25 \%$ to $88 \%(1-3)$.

Nevertheless, surgeons should not forget other possible diseases that might present in the form of a polyp in the EAC. There is an extensive list of diagnoses that also may present as AP and they vary both in prevalence and in gravity and urgency. The frequent apprehension caused by these lesions is that they can be the manifestation of neoplastic lesions. Some examples are paragangliomas, osteochondromas, neuromas, basal cell carcinoma (BCC), squamous cell carcinoma (SCC), Langerhans cell histiocytosis (LCH), and even metastasis from distant organs. Not neoplastic but just as dramatic is the diagnosis of malignant otitis externa (MOE). It should also be kept in mind since it is an infection that should be promptly treated with antibiotics. For its destructive characteristic, it often needs differential diagnosis with malignant neoplasm (4).

In that way, very few papers have been published describing the frequency of the main etiologies of aural polyps. When searching PubMed/MEDLINE for the terms "aural polyp" or "external ear polyp", less than 140 papers are listed.

\section{AIM}

Considering the lack of information on this subject, the objective of this study is to analyze the prevalence of aural polyps and their associated diagnosis in patients referred to the Otology Division of a tertiary hospital. We also aim to evaluate if clinical signs or symptoms could help to identify the etiology correctly.

\section{MATERIALS AND METHODS}

This was a cross-sectional study with patients from a cohort from the Otology Division of a university hospital in south Brazil. Patients were evaluated from July 2001 to December 2020. The inclusion criteria were to have unilateral or bilateral aural polyps at the first consultation and a complete medical chart with the description of imaging exams or surgery (and histology) details, when applicable. Patients with an incomplete online medical chart, with a history of previous ear surgery, or who did not re- turn to follow-up consultation and, consequently, did not receive a final diagnosis, were excluded.

All patients underwent, at the first consultation, an anamnesis for a detailed history of symptoms with a systematic protocol. Complete otorhinolaryngological physical examination was performed, with recorded video-otoscopy of both ears for all patients.

Details about the AP characteristics were collected through the anamnesis protocol, revision of the video-otoscopy and the online medical chart and consisted of:

a. Physical examination details:

$\diamond$ side;

$\diamond$ grade of occlusion in the ear canal (less than $50 \%$, between 50 and $90 \%$, and more than 90\%) and when not completely occlusive, if it was suggestive of COM or not;

$\diamond$ apparent quadrant of origin;

$\diamond$ surface aspect (epithelized or reddish).

b. Associated symptoms:

$\diamond$ ear discharge;

$\diamond$ ear bleeding.

c. Involvement of the contralateral ear (CLE): if normal or which diagnosis.

d. Surgical details:

$\diamond$ intraoperative diagnosis;

$\diamond$ if cholesteatoma, which was the route of formation (posterior mesotympanic, posterior or anterior epitympanic, two-route or indeterminate) as previously described by Rosito et al. (8);

$\diamond$ presence or absence of cholesterol granuloma or granulation tissue;

$\diamond$ state of the facial nerve (preserved or affected).

e. Diagnosis: for patients who did not undergo surgery, results of imaging exams or biopsy were reviewed.

This study was approved by the Hospital de Clínicas de Porto Alegre Research and Ethics Committee (\# 01-431). All participating patients (or their legal guardians when the participant was a child) provided written informed consent before their inclusion in the study. Data analysis was conducted from February 2020 to March 2020. Statistical analysis using the chi-square test was performed using SPSS, version 20.0 (SPSS, Inc). Statistical significance was set at $\mathrm{P}<0.05$. 


\section{RESULTS}

Of the 2432 patients evaluated, $133(5.4 \%)$ had a polyp in the external auditory canal. Fifty-three were excluded, leaving a final sample of 81 ears $(3.3 \%)$ in
78 patients, since 3 patients had bilateral polyps. Of those, $56.8 \%$ were male and the average age of the population studied was 31 years (standard deviation of 18.6) with the oldest patient being 74 and the

Table 1. Prevalence of diagnosis associated with aural polyps

\begin{tabular}{l|c|c} 
Final Diagnosis & $\mathbf{n}$ & \% \\
Middle ear cholesteatoma (MEC) & 47 & 58 \\
Non-cholesteatomatous chronic otitis media (NCCOM) & 23 & 28.3 \\
Squamous cell carcinoma (SCC) & 3 & 3.7 \\
Tympanic paraganglioma & 2 & 2.4 \\
Facial schwannoma & 2 & 2.4 \\
Jugular foramen schwannoma & 1 & 1.2 \\
Endolymphatic sac tumor & 1 & 1.2 \\
Neuroendocrine carcinoma & 1 & 1.2 \\
Myringitis granulosa & 1 & 1.2 \\
Total & 81 & 100 \\
\hline
\end{tabular}

Table 2. Prevalence of diagnosis associated with ear discharge

\begin{tabular}{lc|c|c|} 
& \multicolumn{3}{c|}{ Ear Discharge } \\
& Yes & No & Total \\
MEC & N (\%) & N (\%) & $47(61.8 \%)$ \\
NCCOM & $42(63.3 \%)$ & $5(50 \%)$ & $23(30.3 \%)$ \\
SCC & $19(28.8 \%)$ & $4(40 \%)$ & $2(2.6 \%)$ \\
Tympanic paraganglioma & $2(3 \%)$ & 0 & $1(1.3 \%)$ \\
Facial schwannoma & $1(1.5 \%)$ & 0 & $1(1.3 \%)$ \\
Myringitis granulosa & 0 & $1(10 \%)$ & $1(1.3 \%)$ \\
Endolymphatic sac tumor & $1(1.5 \%)$ & 0 & $1(1.3 \%)$ \\
Total & $1(1.5 \%)$ & 0 & $76(100 \%)$ \\
\hline
\end{tabular}

Table 3. Prevalence of diagnosis associated with ear bleeding

\begin{tabular}{l|c|c|}
\hline \multirow{2}{*}{} & \multicolumn{2}{|c|}{ Ear Bleeding } \\
\cline { 2 - 3 } & Yes & No \\
MEC & $\mathbf{N}(\%)$ & $\mathbf{N}(\%)$ \\
NCCOM & $22(61.1 \%)$ & $19(59.4 \%)$ \\
SCC & $12(33.3 \%)$ & $10(31.3 \%)$ \\
Tympanic paraganglioma & $2(5.6 \%)$ & 0 \\
Facial schwannoma & 0 & $1(3.1 \%)$ \\
Myringitis granulosa & 0 & $1(3.1 \%)$ \\
Total & 0 & $1(3.1 \%)$ \\
\hline
\end{tabular}


Alice Lang Silva, Fábio André Selaimen, Isabel Saorin Conte et al..

youngest being 1 year old. Regarding side, 46.9\% $(n=38)$ of aural polyps were on the right ear. The frequencies of the final diagnosis of the total sample are described in Table 1:

Ear bleeding was present in $81.4 \%(n=66)$ of ears and the most frequent etiologies were cholesteatoma (63.6\%) and COM without cholesteatoma (28.8\%). Ear discharge was also observed in one case of tympanic paraganglioma (Table 2).

Previous history of ear bleeding was present in $44.4 \%$ of patients $(\mathrm{n}=36)$ and a pulsatile characteristic in $7.4 \%(\mathrm{n}=6)$. Most polyps had a reddish aspect $(79 \%, \mathrm{n}=64)$ and the remainders had either an epithelialized surface $(16 \%, n=13)$ or were not well characterized on the medical chart $(5 \%, n=4)$. Ear bleeding was observed in most etiologies, according to Table 3 .

The history of bleeding was also similar between reddish or epithelialized polyps $(\mathrm{p}=1)$ and $50 \%$ of pulsatile polyps had a history of bleeding (Table 4).

The grade of occlusion was complete in $65.4 \%$ of patients $(n=53)$, partial in $13.5 \%(n=11)$ and intermediate (between $50-90 \%$ ) in $19.7 \%$ of the patients $(\mathrm{n}=16)$. From patients who did not have a complete occlusion of the EAC $(n=27)$, it was possible to determine the apparent quadrant of origin as: inferior
$(8.7 \%, \mathrm{n}=7)$, superior $(13.7 \%, \mathrm{n}=11)$, posterior $(10 \%$, $\mathrm{n}=8)$, and anterior $(1.2 \%, \mathrm{n}=1)$. In 20 of those, it was possible to make an initial hypothesis of chronic otitis media from the video-otoscopy.

Concerning MEC, the frequency of the routes of formation was: 12 posterior epitympanic, 12 posterior mesotympanic, 7 two-routes (both the pars flaccida and the pars tensa are involved), and 10 undetermined. Another case was classified as congenital and five patients had not undergone surgery yet (Table 5).

When analyzing the contralateral ear (CLE) of the polyp, we found that the half of them had a normal otoscopy $(50.6 \%, n=41)$. From the remainder, the most common diagnosis was that of tympanic membrane (TM) retraction $(17.2 \%, \mathrm{n}=14)$, followed by middle ear cholesteatoma $(14.8 \%, \mathrm{n}=12)$, TM perforation (11.1, $\mathrm{n}=9)$, and another aural polyp (3.7\%, $\mathrm{n}=3)$. We have not observed a statistical difference $(\mathrm{p}=0.77)$ in the prevalence of CLE alterations between polyps from COM (50.7\%) and other etiologies (40.0\%). In patients whose polyps were associated with cholesteatoma, the alterations in the CLE were: another cholesteatoma in $17 \%(n=8), C O M$ without cholesteatoma in $14.8 \%(\mathrm{n}=7)$, TM retraction in $17 \%(\mathrm{n}=8)$, and another polyp in 3 of them (all the patients who had bilateral polyps in our sample).

Table 4. Prevalence of visual characteristic of aural polyps associated with ear bleeding

\begin{tabular}{l|c|c|c|} 
& \multicolumn{3}{|c|}{ Ear Bleeding } \\
\cline { 2 - 4 } & Yes & No & Total \\
Epithelialized & $\mathbf{N}(\%)$ & $\mathbf{N}(\%)$ & $\mathbf{N}(\%)$ \\
Reddish & $6(50 \%)$ & $6(50 \%)$ & $12(100 \%)$ \\
Total & $30(53.6 \%)$ & $26(46.4 \%)$ & $56(100 \%)$ \\
\hline
\end{tabular}

Table 5. Route of formation of COM with cholesteatoma

\begin{tabular}{lcc} 
Cholesteatoma Classification - Routes of Formation & n & $\%$ \\
Posterior epitympanic & 12 & 25.5 \\
Posterior mesotympanic & 12 & 25.5 \\
Two-routes & 7 & 14.8 \\
Undetermined & 10 & 21.2 \\
Congenital & 1 & 2.1 \\
Awaiting surgery & 5 & 10.6 \\
Total & 47 & 100 \\
\hline
\end{tabular}


The final diagnosis was established through biopsy in 6 patients (7.4\%). Through clinical examination and radiological assessment in 12 (14.8\%)-after proper cauterization along with careful and detailed cleaning of the ear canal in more than one follow-up visit, some polyps decreased and the diagnosis could be made through otoscopy, and through surgery in 62 (76.5\%). Biopsy resulted in one neuroendocrine carcinoma, two non-cholesteatomatous COM (NC$\mathrm{COM}$ ), which refers to all other forms of COM such as TM perforations and retractions, and three squamous cell carcinomac (SCC). Clinical and radiological assessment allowed the diagnosis of 5 cases of MEC, 5 cases NCCOM, 1 case of myringitis granulosa, and 1 case of a facial schwannoma. Even though 62 diagnoses were only established through surgery, a total of 66 patients went through surgery for treatment purposes.

\section{DISCUSSION}

The etiologies behind aural polyps in our sample were quite varied when comparing with data from previous studies. Nevertheless, as expected, the most common diagnosis was of different subtypes of COM, with cholesteatoma as the leading cause.

The prevalence of cholesteatoma obtained in this study was $58 \%$, which is in accordance with Milroy et al. (9), López Aguado et al. (10), and Arroyo Gargallo et al. (11) who observed prevalence of cholesteatoma in patients with polyps of $43.7 \%, 50 \%$, and $52 \%$, respectively. Other authors, such as Xenellis et al. (7) and Kalra et al.(2) obtained a higher prevalence of cholesteatomas: $86.7 \%$ and $88 \%$, respectively. All these studies were retrospective and subject to several biases inherent to the nature of this study design. Nevertheless, all studies demonstrate that most polyps in the external auditory often can hide a cholesteatoma.

Regarding subtypes of cholesteatoma, our Otology Division was responsible for the classification described previously in the literature by Rosito et al. (8) and we systematically classify cholesteatomas through otoscopy and during surgery. The frequency of occurrence of each route of cholesteatoma formation in patients with polyps had never been demonstrated before in the literature, to our knowledge. In this study, it can be seen that there is no prevalence of any specific type in patients with aural polyps. Also, the prevalence of each subtype follows the distribution seen in the general population of patients with cholesteatoma (8).

Curiously, granulation tissue had a low prevalence in the middle ear and the mastoid based on surgery descriptions. Considering that polyps are usually an inflammatory mass of granulation, we expected more findings of inflammatory tissue during surgeries. As such, cholesterol granuloma was described in a small percentage of surgeries. It was also possible to determine that polyps were not associated with greater involvement of the facial nerve, since it was only involved in $6(7.4 \%)$ cases in this sample, and in 4 of them, the underlying disease was a neoplasm. This sign was also associated with malignancy according to Xenellis et al. (7) and also had a low prevalence according to Kalra et al. (2).

Diseases different than cholesteatoma were represented with a lower frequency, of them, other forms of COM were the most frequent. These data are in agreement with those obtained by other authors $(2,4,7)$ whose study showed that neoplasms and infectious causes were less frequent diagnoses. In fact, we had no cases of malignant otitis externa in our sample, even though it was a condition previously described as an important differential diagnosis by other authors (4). This may be because our outpatient clinic only receives patients by referral, and patients with MOE usually seek the emergency room.

Prior studies have described different distributions of rarer diseases that may manifest as polyps in EAC (2-7). Some of these were also found in our sample, such as paragangliomas. The importance of establishing the prevalence of each disease as the underlying cause of an $\mathrm{AO}$ is that it allows us to better interpret the results of imaging exams. Based on the prevalence, a better pre-test estimation can be made-never putting aside a careful anamnesis and physical examination. History and time of symptoms usually gives a good initial hypothesis since patients with COM who develop a polyp usually do so after year-long disease duration. However, our study has found no association between specific clinical symptoms, such as ear discharge or bleeding, and any specific diagnosis.

For this reason, a preoperative diagnosis with computed tomography (CT), magnetic resonance 
Alice Lang Silva, Fábio André Selaimen, Isabel Saorin Conte et al..

imaging (MRI), and even biopsy should always be attempted. When a diagnosis can be made preoperatively, it not only guides treatment (which differs according to the underlying disease and stage) but also prevents unnecessary risks, such as a biopsy of a vascular lesion, and helps to identify potential challenges in the operating room (OR).

\section{CONCLUSION}

Chronic otitis media with cholesteatoma is the variation most associated with polyps. However, there is an interesting list of other etiologies that may be involved, such as benign and malignant neoplasms, which require great care before deciding on an intervention. Our study shows that symptoms associated with polyps and their aspect do not reveal the most probable etiologies making imaging exams, biopsy and surgery necessary steps in aural polyp investigation.

Author contributions: All authors have given substantial contributions to the concept and design, acquisition of data, or analysis and interpretation of data; F. A. Selaimen, A. L. Silva, S. S. Costa, and L. P. S. Rosito worked on drafting the article or revising it critically for important intellectual content; and all authors contributed to the final approval of the version to be published.

Conflict of interest: Nothing to declare.

Funding: This research did not receive any specific grant from funding agencies in the public, commercial, or not-for-profit sectors.

\section{REFERENCES}

6. Tay HL, Hussain SS. The management of aural polyps. J Laryngol Otol. 1997;111(3):212-4. doi: 10.1017/ s002221510013693x.

7. Kalra VK. Aural polyp is not always due to chronic otitis media (COM): Preoperative computed tomographic scan is good pointer for sinister lesions. Indian J Otolaryngol Head Neck Surg. 2018;70(4):505-9. doi: 10.1007/s12070-018-1482-5.

8. Brea B, Roldán Fidalgo A. Diagnóstico por imagen de las lesiones benignas del conducto auditivo externo [Imaging diagnosis of benign lesions of the external auditory canal]. Acta Otorrinolaringol Esp. 2013;64(1):6-11. Spanish. doi: 10.1016/j. otorri.2012.06.004.
9. Spielmann PM, McKean S, White RD, Hussain SS. Surgical management of external auditory canal lesions. J Laryngol Otol. 2013;127(3):246-51. doi: $10.1017 /$ S0022215112003155.

10. Veitch D, Brockbank M, Whittet H. Aural polyps and cholesteatoma. Clin Otolaryngol Allied Sci. 1988;13(5):395-7. doi: 10.1111/j.1365-2273.1988. tb00772.x.

11. Gliklich RE, Cunningham MJ, Eavey RD. The cause of aural polyps in children. Arch Otolaryngol Head Neck Surg. 1993;119(6):669-71. doi: 10.1001/ archotol.1993.01880180089016.

12. Xenellis J, Mountricha A, Maragoudakis P, Kandiloros D, Assimakopoulos D, Linthicum FJ, Nikolopoulos TP. A histological examination in the cases of initial diagnosis as chronic otitis media with a polypoid mass in the external ear canal. Auris Nasus Larynx. 2011;38(3):325-8. doi: 10.1016/j. anl.2010.10.002.

13. Rosito LS, Netto LF, Teixeira AR, da Costa SS. Classification of cholesteatoma according to growth patterns. JAMA Otolaryngol Head Neck Surg. 2016;142(2):168-72. doi: 10.1001/jamaoto.2015.3148.

14. Milroy CM, Slack RW, Maw AR, Bradfield JW. Aural polyps as predictors of underlying cholesteatoma. J Clin Pathol. 1989;42(5):460-5. doi: 10.1136/ jcp.42.5.460.

15. López Aguado D, López Campos D, Pérez Piñero B, Campos Bañales ME. El pólipo aural en la patología inflamatoria crónica del oído medio [Aural polyp in chronic inflammatory middle ear disease]. Acta Otorrinolaringol Esp. 2003;54(3):161-4. Spanish. doi: 10.1016/s0001-6519(03)78400-3.

16. Arroyo Gargallo R, Herráiz Puchol C, Santos Santos S, Olaizola Gorbea F. Pólipos de CAE y colesteatoma [Polyps of the external auditory canal and cholesteatoma]. Acta Otorrinolaringol Esp. 1997 Aug-Sep;48(6):447-51. Spanish.

17. Rosito LS, Netto LS, Teixeira AR, da Costa SS. Sensorineural Hearing Loss in Cholesteatoma. Otol Neurotol. 2016;37(3):214-7. doi: 10.1097/ MAO.0000000000000952. 